\title{
Phase Distortion Corrections of Phase-Only LCoS Devices for Digital Holographic Applications
}

\author{
Yuan Tong, Mike Pivnenko, and Daping Chu* \\ Centre for Photonic Devices and Sensors, Department of Engineering, University of Cambridge, 9 JJ Thomson Avenue, Cambridge CB3 OFA, \\ United Kingdom \\ *Corresponding author:dpc31@cam.ac.uk
}

\begin{abstract}
Significant phase distortion corrections of nearly perfect phase linearity and minimum phase flicker with doubled meaningful phase levels was achieved by optimising the digital driving patterns of phase-only LCoS devices for digital holographic applications.
\end{abstract}

OCIS codes: (090.1995) Digital Holography; (070.6120) Spatial Light Modulator; (230.3720) Liquid-Crystal Devices

\section{Introduction}

Digital holography (DH) has a wide range of applications, such as digital holographic microscopy (DHM) to extract the 3D information of a biological organism or a physical object by a single recorded hologram [1]. Liquid crystal on silicon spatial light modulators (LCoS SLM) can be employed in the DHM system to either control and shape the sample illumination, or act as spatial Fourier filters in the imaging path [2]. However, when using LCoS devices, it is unavoidable to have phase distortions in terms of phase linearity and phase stability, i.e. phase flicker, which could introduce unnecessary depth distortion and noise resulting in a loss of resolution in tomography measurements.

Ideally, the phase response is expected to be linear with a range of at least from 0 to $2 \pi$ for the operating wavelength. However, the orientation of liquid crystal (LC) molecules does not follow a linear behaviour in relation to the applied voltage, which results in a nonlinear control of the phase depth. For commercial LCoS devices, the linearisation process can be done by gamma correction in software.

Phase flicker, which can be profound in digital driving LCoS devices, introduces instability in the applied phase, causing the reduction in resolution. The digital driving pattern forces the LC molecules and produces certain temporal fluctuations, resulting in a fast-changing phase shift error. Drifting around the desired phase value makes it difficult to resolve the adjacent phase levels, and hence reduces the phase and hence depth resolution that can be achieved.

Some previous studies that have tried to minimise the phase flicker include: Martínez et al. reasonably reduced the flicker by using a higher frequency for the driving sequence [3]; García-Márquez et al. demonstrated a reduction of up to $80 \%$ of the flicker initial value by bringing the $\mathrm{LCoS}$ device to $-8^{\circ} \mathrm{C}$ [4]. Another technique reported to reduce the phase fluctuations was based on a polarisation state generator, which was configured to reduce the degree of depolarisation on the light beam reflected by LCoS and diattenuation [5]. However, these methods suffer from either a slow switching speed of the $\mathrm{LCoS}$ devices or the presence of a large remaining flicker.

This work goes back to the molecular level behaviour of the LCs and attempts to correct the phase distortions from the point of view of fundamental mechanism. A novel method is proposed to linearise the phase depth and minimise the phase flicker at the same time by optimising the driving patterns, based on the existing digital driving method.

\section{Phase Distortion Corrections}

A crossed-polariser based system was set up to characterise the phase distortions in phase-only LCoS device, which was assembled in the group. With the laser beam shining at a fixed point of the LCoS active area, output intensities were recorded when 256 bitmaps (with 0-255 different grey levels) were displayed on the LCoS in turn. Corresponding phase responses could then be derived by Equation (1), where $I_{\text {nor }}$ is the normalised intensity.

$$
\Delta \emptyset=2 \sin ^{-1} \sqrt{I_{\text {nor }}}
$$

\subsection{Phase Linearisation}

Since the applied voltage levels are mapped as grey levels, the phase depth was linearised with respect to the grey level by opting the driving patterns which could lead to a linear response. The linearity is only guaranteed within the driving voltage range where the linearisation is performed, i.e. $1.0 \mathrm{~V}$ to $2.7 \mathrm{~V}$ in this case.

Fig. 1(a) shows the normalised intensity profiles obtained directly from the characterisation system. One peak point and one valley point can be observed in the sinusoidal intensity curve, where the difference of the phase depth between them is $\pi$. Fig. 1(b) illustrates the corresponding phase curves. Nearly perfect linearity can be noted within the phase range of 0 to $2.6 \pi$ after the linearisation. 

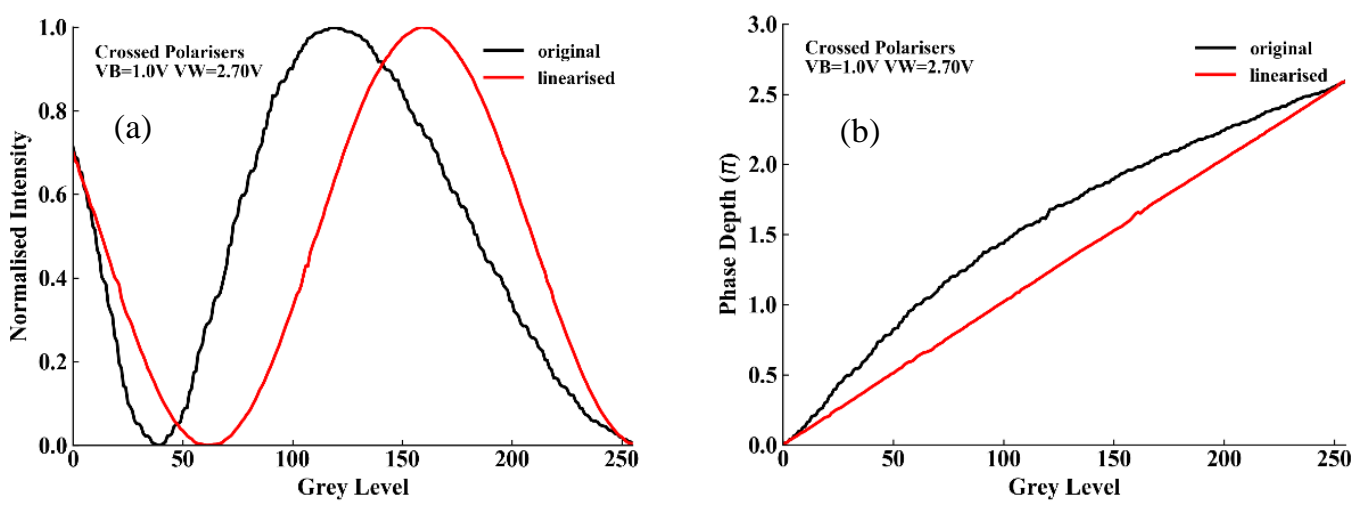

Fig. 1 (a) Normalised intensity profiles as measured; (b) Phase depth profiles before and after linearization.

\subsection{Phase Flicker Minimisation}

Digital driving patterns at each grey levels was optimised to achieve the desired low-level phase flicker by selecting suitable ones among tens of millions of possible pulse arrangements.

Fig. 2(a) shows the normalised intensity profiles and their peak-to-peak intensity profiles over time at each grey level (hy and ty refer to the initial optimised driving patterns and the final optimised driving patterns, respectively). The small oscillations in the peak-to-peak intensity profiles are the direct results of the driving patterns with different pulse arrangement. Fig. 2(b) shows the comparison of the standard deviation of the phase, i.e. the phase flicker, in degree at each grey level. As the intensity approaches the minimum and maximum, the little change in intensity combined with an obvious change in phase results in the corresponding phase flicker reaches its highest value. With further optimization, the phase flicker has been evidently reduced to half of its previous value, i.e. mostly below $1^{\circ}$. Therefore the meaningful phase levels, i.e. phase step larger than phase flicker, over $2 \pi$ can now be doubled from 256 steps ( 8 bits) to 512 steps ( 9 bits).
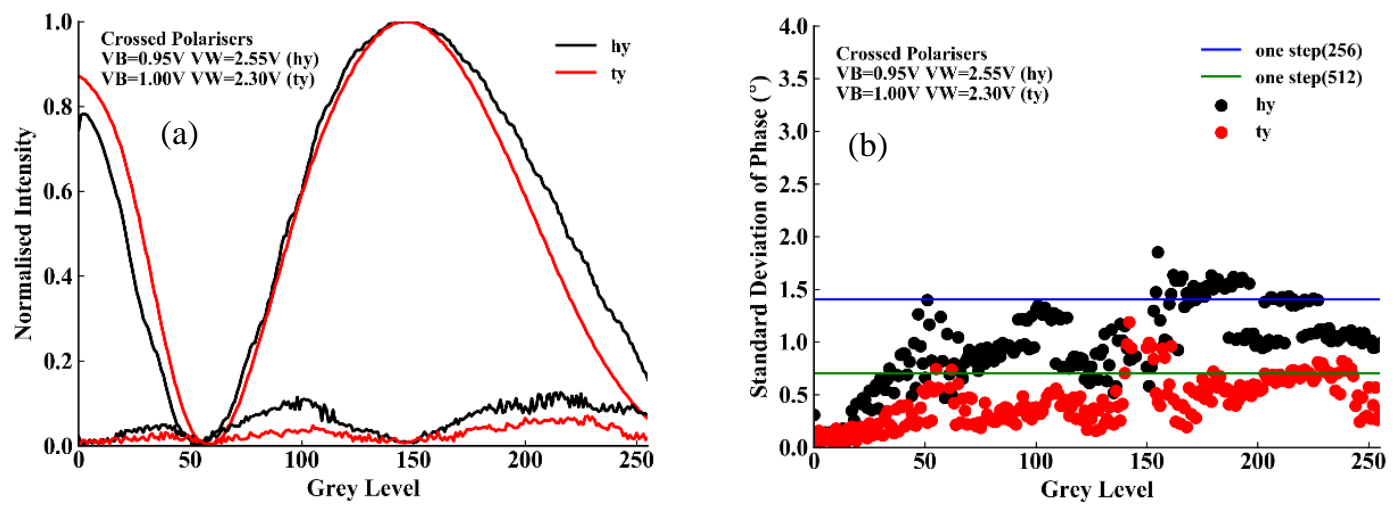

Fig.2 (a) Normalised intensity profiles as measured and their peak-to-peak intensity profiles; (b) corresponding phase flickers.

\section{Conclusion}

Phase distortion generated by phase-only LCoS devices have been corrected to linearise the phase depth and minimise the phase flicker by optimising the digital driving patterns. Nearly perfect linearity has been achieved for a specific driving voltage range, and the phase flicker has been halved to have a meaningful doubling of the phase grey scales, enabling a $50 \%$ increase in the depth resolution for DHM system.

\section{References}

[1] W. Osten et al., "Recent advances in digital holography [Invited]," Appl. Opt., vol. 53, no. 27, p. G44, 2014.

[2] C. Maurer, A. Jesacher, S. Bernet, and M. Ritsch-Marte, "What spatial light modulators can do for optical microscopy," Laser Photonics Rev., vol. 5, no. 1, pp. 81-101, 2011.

[3] F. J. Martínez, A. Márquez, S. Gallego, J. Francés, and I. Pascual, "Extended linear polarimeter to measure retardance and flicker: application to liquid crystal on silicon devices in two working geometries," Opt. Eng., vol. 53, no. 1, p. 014105, Jan. 2014.

[4] J. García-Márquez et al., "Flicker minimization in an LCoS spatial light modulator," Opt. Express, vol. 16, no. 8, pp. 16711-16722, 2008.

[5] A. Márquez, I. Moreno, C. Iemmi, A. Lizana, J. Campos, and M. J. Yzuel, "Mueller-Stokes characterization and optimization of a liquid crystal on silicon display showing depolarization.," Opt. Express, vol. 16, no. 3, pp. 1669-1685, Feb. 2008. 